\title{
Speed Identification Method of Induction Motor Based on Improved MRAS
}

\author{
Shi Xiaorong \\ Hunan Institute of Engineering, Hunan China, 411101 \\ 562744559@qq.com
}

\begin{abstract}
Aiming at the traditional model reference adaptive control system (MRAS) in the estimation of low speed and zero speed regions is not accurate and the performance of MRAS is poor. So it proposes a new method to estimate the speed of induction motors. It uses two types of errors at the same time: one is the error between rotor fluxes, another is the error of electromagnetic torque. It is used to improve the performances of sensorless vector control system at low and zero speed zone. It uses Proportional-integral controller and lowpass filter to constitute a new adaptive mechanism, and combines with Lyapunov approach to design controller. Simulation and experiment results show the effectiveness of the proposed speed estimation method at low and zero speed regions, and good robustness with respect to parameter variations, measurement errors, and noise is obtained.
\end{abstract}

Keywords: Induction motor; Model reference adaptive system (MRAS); Speed estimation; Lyapunov method

\section{NOMENCLATURE}

$\psi_{r m} 、 \psi_{r t}:$ M-T axis rotor flux linkages $\quad \psi_{r d} 、 \psi_{r q}:$ d-q axis rotor flux linkages

$\psi_{r}:$ Total rotor flux vector

$T_{e}:$ Electromagnetic torque

$T_{L}:$ Load torque

$J_{p}:$ Moment of inertia

$f$ : Coefficient of viscous

$n_{p}:$ Number of pole pairs

$\omega_{r}:$ Rotor speed $(\mathrm{rad} / \mathrm{s})$

$\omega_{s}:$ Slip angular frequency $(\mathrm{rad} / \mathrm{s})$

$T_{r}:$ Rotor time-constants $\left(T_{r}=\frac{L_{r}}{R_{r}}\right.$ ) $\sigma$ : Leakage flux total coefficient $\left(\sigma=1-\frac{L_{m}^{2}}{\left.L_{s} L_{r}\right)}\right.$

$\varphi_{r}:$ Rotor flux linkage angle

$R_{s} 、 L_{s} 、 L_{m} 、 L_{r}:$ Stator resistances, stator inductances, mutual inductance, rotor inductances

\section{Introduction}

For electric vehicles with induction motor vector control system [10], the speed of information is required. Speed may be measured by sensors or estimated by the voltage signals, current signals and the motor parameters. However, using sensors to measure the rotor speed will bring a series of questions, such as: speed sensor is not only costly, but also brings a lot of inconveniences to install. The installation accuracy of speed sensor will affect the accuracy of speed measurement directly, there are some difficulties to maintenance the 
induction motor, and it destroyed the features of motor undermine. Under relatively harsh conditions, the measurement accuracy of the speed sensor is also vulnerable. Promotion and application of induction motor may cause some impacts if install the speed sensor. Speed sensorless drive control system becomes one of the important research directions of electric vehicles [1].

It has been proposed several methods to estimate the speed [2] for speed sensorless vector control system of induction motor. These methods can be divided into two categories: signal injection-based estimator and the model-based observer. The first category includes: model reference adaptive control system (model reference adaptive system, MRAS)[3-5], the adaptive Luenberger observer and the extended Kalman filter. The main disadvantages of model-based observer are their insufficient performance at low speeds and parameters machine sensitivity. In order to overcome these shortcomings, the signal injection methods were proposed. Although these methods have good performance at low and zero speed region, they are complex and required external hardware signal injection and the adverse effect of injecting signal on the machine performance. Therefore, the model-based methods and especially MRAS-based methods are the most widely used until now due to its simplicity.

Many MRAS methods are proposed based on the rotor flux, back-EMF, reactive power

and voltage - current [6] $\left(u_{s} \otimes i_{s}\right)$ signals of stator. The model-based of rotor flux first proposed by Schauder [8], still remain the most widely used methods, and did a number of studies to improve its performance. Indeed, the disadvantages of this method are parameter (especially the stator resistance) sensitivity and pure integration problems limit the motor performance at low and zero speed region.

It has been proposed many ways to improve motor performance in the zero speed region, including: online identification of stator resistance, using MRAS while estimating speed and stator resistance. And in order to overcome the difficulties of pure integrator, a low cut-off frequency programmable low-pass filter cascade (programmable cascaded low-pass filter, LPF) [9] were proposed to instead of pure integration section. This paper proposes a method based on two errors of the rotor flux and the electromagnetic torque, use a low-pass filter to instead of pure integration and increase the estimated stator current feedback compensation to constitute MRAS speed estimation. This method is used in sensorless vector control system of induction motor electric vehicle. Building corresponding simulation models in Matlab / Simulink to verify its performance. Finally, this method was used in $5 \mathrm{~kW}$ induction motor of electric vehicles speed sensorless vector control system to do experimental verification. The results show that this method improves the performance of wide speed range estimation, especially at low and zero speed region, obtained a very good control effect.

\section{Vector Control Mode of Induction Motor}

Rotor flux oriented vector control system [9] can achieve full decoupling of current without increasing the decoupling controller, and there is no static stability limit condition. The control methods are simple and have good dynamic performance and control accuracy. Using this control method can obtain the same linear mechanical properties with DC motor. So it is widely used by the asynchronous motor in rotor flux oriented vector control system.

The goal of rotor flux oriented vector control is to make the stator current decoupling of flux and torque component, which can be controlled and adjusted respectively to get a good dynamic performance. When the two-phase synchronous rotating coordinate system was orientated according to rotor flux, the flux defined in the rotor reference frame as:

$$
\psi_{r d}=\psi_{r m}=\psi_{r}, \psi_{r q}=\psi_{r t}=0
$$

The voltage state equations of induction motor expressed in the synchronous reference frame are: 


$$
\left\{\begin{array}{l}
u_{s}=R_{s} i_{s}+\sigma L_{s} \frac{d i_{s}}{d t}+\frac{L_{m}}{L_{r}} \frac{d \psi_{r}}{d t}+j \sigma L_{s} \omega_{s} i_{s}+j \frac{L_{m}}{L_{r}} \omega_{s} \psi_{r} \\
0=\frac{1}{T_{r}} \psi_{r}-\frac{L_{m}}{T_{r}} i_{s}+\frac{d \psi_{r}}{d t}+j \omega_{r} \psi_{r}
\end{array}\right.
$$

The motion equation of the system is:

$$
T_{e}-T_{L}=\frac{J_{p}}{n_{p}} \frac{d \omega_{r}}{d t}+f \omega_{r}
$$

The electromagnetic torque equation is:

$$
T_{e}=n_{p} \frac{L_{m}}{L_{r}}\left(i_{s q} \psi_{r d}-i_{s d} \psi_{r q}\right)
$$

If we assume that the torque load and the viscous coefficient are unknown, and consider that $T=T_{L}+f \omega_{r}$, we can write:

$$
T_{e}-T=\frac{J_{p}}{n_{p}} \frac{d \omega_{r}}{d t}
$$

Using equation (1) into equation (4), the rotor flux oriented electromagnetic torque can be rewritten as:

$$
T_{e}=k_{1} \psi_{r} i_{s q}
$$

Where $k_{1}=\frac{n_{p} L_{m}}{L_{r}}$, and other vector control equations are:

$$
\left\{\begin{array}{l}
\omega_{s}=\frac{L_{\mathrm{m}} i_{s t}}{T_{r} \psi_{r}} \\
\psi_{r}=\frac{L_{\mathrm{m}}}{T_{r} p+1} i_{s m} \\
\varphi_{r}=\int\left(\omega_{s}+\omega_{r}\right) d t
\end{array}\right.
$$

\section{MRAS Speed Estimator}

The error signals using MRAS method to estimate speed can be divided into: the rotor flux, back-EMF and reactive power. The accuracy of the rotor flux based MRAS speed observer [8] depends on the matching degree of rotor flux observer and the actual rotor flux of motor.

\subsection{Classical MRAS Speed Estimator}

Voltage equation of induction motor (2) can be changed into the stator reference frame as:

$$
\left\{\begin{array}{l}
u_{s}=R_{s} i_{s}+\sigma L_{s} \frac{d i_{s}}{d t}+\frac{L_{m}}{L_{r}} \frac{d \psi_{r}}{d t} \\
0=\frac{1}{T_{r}} \psi_{r}-\frac{L_{m}}{T_{r}} i_{s}+\frac{d \psi_{r}}{d t}-j \omega_{r} \psi_{r}
\end{array}\right.
$$


When using traditional MRAS methods to estimate the rotot speed, according to equation (8), the voltage model equation and current model [12] equation of rotor flux can be written as:

$$
\begin{aligned}
& \psi_{r v}=\frac{\sigma L_{s} L_{r}}{L_{m}} i_{s}+\frac{L_{r}}{L_{m}} \int\left(u_{s}-R_{s} i_{s}\right) d t \\
& \psi_{r i}=\int\left(\frac{L_{m}}{T_{r}} i_{s}-\left(\frac{1}{T_{r}}-j \omega_{r}\right) \psi_{r i}\right) d t
\end{aligned}
$$

According to the MRAS principle, for the same input signal, the current model equation can be written in an estimated form as:

$$
\hat{\psi}_{r i}=\int\left(\frac{L_{m}}{T_{r}} i_{s}-\left(\frac{1}{T_{r}}-j \hat{\omega}_{r}\right) \hat{\psi}_{r i}\right) d t
$$

Where $\hat{x}\left(x=\psi_{r \alpha}, \psi_{r \beta}, \omega\right)$ are adjustable estimation parameters.

The dynamic equation of the estimation error can be obtained by subtracting (10) and (11): $e_{\psi}=\psi_{r i}-\hat{\psi}_{r i}$, and

$$
e_{\psi}^{\prime}=-\left(\frac{1}{T_{r}}-J \omega_{r}\right) e_{\psi}+J\left(\omega_{r}-\hat{\omega}_{r}\right) \hat{\psi}_{r}
$$

The main purpose of the MRAS method is to ensure the system (12) is stable, which naturally requires the error closes or equals to zero. Using the hyper-stability Popov theory does some stability studies of this algorithm. The derivation of the error signal is mainly composed of two parts, one part is linear and another part is non-linear.

The differential equation (12) can be written in the form of a matrix as:

$$
e_{\psi}^{\prime}=A e_{\psi}-W
$$

Where $A=-\frac{1}{T_{r}} I+J \omega ; W=J\left(\omega_{r}-\hat{\omega}_{r}\right) \hat{\psi}_{r}=J \Delta \omega_{r} \hat{\psi}_{r}$;

$$
e_{\psi}=\left[\begin{array}{l}
\psi_{r \alpha}-\hat{\psi}_{r \alpha} \\
\psi_{r \beta}-\hat{\psi}_{r \beta}
\end{array}\right] ; I=\left[\begin{array}{cc}
1 & 0 \\
0 & 1
\end{array}\right] ; J=\left[\begin{array}{cc}
0 & -1 \\
1 & 0
\end{array}\right] \text {. }
$$

A is a Hurwitz matrix. According to the Lyapunov function of the linear part: $e_{\psi}^{\prime}=A e_{\psi}$,we can get:

$$
A=e_{\psi}^{T} e_{\psi}
$$

So the derivative of the Lyapunov function be

$$
V^{\prime}=e_{\psi}^{T^{\prime}} e_{\psi}+e_{\psi}^{T} e_{\psi}^{\prime}=e_{\psi}^{T}\left(A^{T}+A\right) e_{\psi}=-\frac{2}{T_{r}} e_{\psi}^{T} e_{\psi}<0
$$

Although the adaptive mechanisms can be derived by Lyapunov function, extracted from the hyper-stability Popov [7] criterion is simpler relatively. According to the Popov criterion, we can be obtained:

$$
\omega=K_{p}\left(\psi_{r} \otimes \hat{\psi}_{r}\right)+K_{i} \int\left(\psi_{r} \otimes \hat{\psi}_{r}\right) d t
$$

Where $K_{p}>0, K_{i}>0$. This adaptive law has obvious problems in the pure integration and DC offset. Therefore, in order to improve their estimated accuracy, many researchers have 
proposed using a low-pass filter LPF to instead of pure integration part. For traditional MRAS speed estimator, its main drawback is that the estimated accuracy is low at low-speed region and the stator resistance has a great influence on voltage model. So the speed estimation method of improved MRAS is proposed to improve its estimated accuracy at low and zero speed region.

\subsection{Improved MRAS Speed Estimator}

In the improved MRAS method, there are two different error signals are used simultaneously, one error is between the rotor fluxes and another error is between the electromagnetic torques. The electromagnetic torque is expressed as equation (4). According to the principle of MRAS, the estimated electromagnetic torque can be expressed as:

$$
\hat{T}_{e}=n_{p} \frac{L_{m}}{L_{r}}\left(I_{s} \otimes \hat{\psi}_{r}\right)
$$

Where $\psi_{r}, \hat{\psi}_{r}$ are given by (9) and (10).

It is well known that the equation of motion(3) governs the mechanical dynamics part of the machine, and the changes of load will result in the change of speed until the electromagnetic torque equal to the load torque. So, based on the same principle, the changes of estimated torque will lead to the changes of estimated speed until the estimated torque is equal to the electromagnetic torques ${ }^{[13]}$. Then the equation of motion (2) can be replaced by the estimated electromagnetic torque and speed as:

$$
\hat{T}_{e}-T_{L}=\frac{J_{p}}{n_{p}} \frac{d \hat{\omega}_{r}}{d t}+f \hat{\omega}_{r}
$$

The dynamics equation of error can be obtained by subtracting (19) from (2):

$$
e_{T}=T_{e}-\hat{T}_{e}=\frac{J_{p}}{n_{p}} \frac{d\left(\omega_{r}-\hat{\omega}_{r}\right)}{d t}+f\left(\omega_{r}-\hat{\omega}_{r}\right)
$$

In order to obtain a good estimated performance of the system, we must consider the following two conditions:

$$
\left\{\begin{array}{l}
e_{\psi}^{\prime}=-\left(\frac{1}{T_{r}}-J \omega_{r}\right) e_{\psi}+j\left(\omega_{r}-\hat{\omega}_{r}\right) \hat{\psi}_{r} \\
e_{T}=T_{e}-\hat{T}_{e}=\frac{J_{p}}{n_{p}} \frac{d\left(\omega_{r}-\hat{\omega}_{r}\right)}{d t}+f\left(\omega_{r}-\hat{\omega}_{r}\right)
\end{array}\right.
$$

According to the same method that determinate the adaptation law of the traditional MRAS and consider the error of torque and speed to determine the new MRAS adaptive control law. So the estimated speed equation becomes:

$$
\hat{\omega}=\left(K_{p}+\frac{K_{i}}{\rho}\right)\left(\psi_{r} \otimes \hat{\psi}_{r}\right)+K_{T} \frac{e_{T}}{\tau \rho+1}
$$

Where: $\tau$ is a constant which closes to the constant of mechanical time. In the principle of improved MRAS speed estimator, the electromagnetic torque error is:

$$
e_{T}=T_{e}-\hat{T}_{e}
$$


By using LPF filter, increasing the feedback compensation of the estimated stator current to improve the accuracy of rotor flux, adding the appropriate adaptive closed-loop control law to get the improved MRAS speed estimator model is shown in Figure 1.

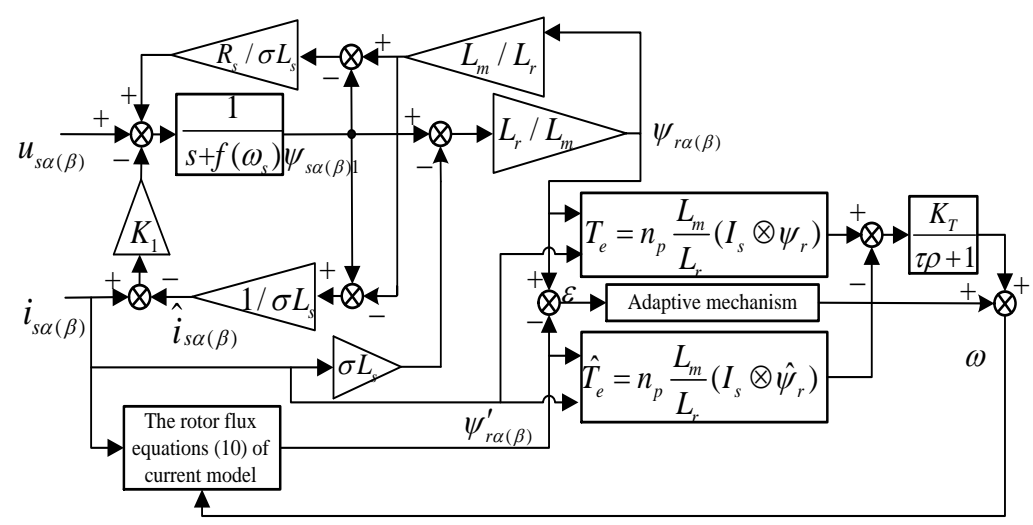

Figure 1. Improved MRAS Speed Estimation Model Diagram

\section{Simulation Analysis}

Figure 2 is a block diagram of induction motor speed sensorless vector control system for electric vehicles based on MRAS [14]. In order to control the induction motor over the entire speed range, it uses weak magnetic controller to obtain accurate rotor flux value. Building simulations of traditional and improved MRAS speed estimator of sensorless indirect vector control system for three-phase induction motor respectively. The simulation parameters are induction motor's parameters measured of electric vehicle experiment platform based on DSP TMS320F2812. These parameters are: rated Power $P_{N}=5 \mathrm{~kW}$, rated line current $I_{N}=75 \mathrm{~A}$, rated line voltage $U_{N}=51 \mathrm{~V}$, rated frequency $f_{N}=100 \mathrm{~Hz}$, rated speed $n_{N}=2850 \mathrm{rad} / \mathrm{min}$, number of pole pairs is 2,Y-shaped connection, stator resistance $R_{s}=0.03186 \Omega$, inductance $L_{s}=0.117 \times 10^{-3} \mathrm{H}$, converted rotor resistance $R_{r}=0.02358 \Omega$, inductance $L_{r}=0.163 \times 10^{-3} \mathrm{H} \quad$, mutual inductance $L_{m}=4.292 \times 10^{-3} \mathrm{H}$.Parameters of SVPWM modulation mode is set to: DC voltage $U_{d c}=72 \mathrm{~V}, T_{P W M}=0.0002 \mathrm{~s}$, the parameters of simulation are set to: simulation step size is ode23, tolerance is 0.001 , the simulation time is $1.5 \mathrm{~s}$. The overall simulation model is shown in Figure 3.

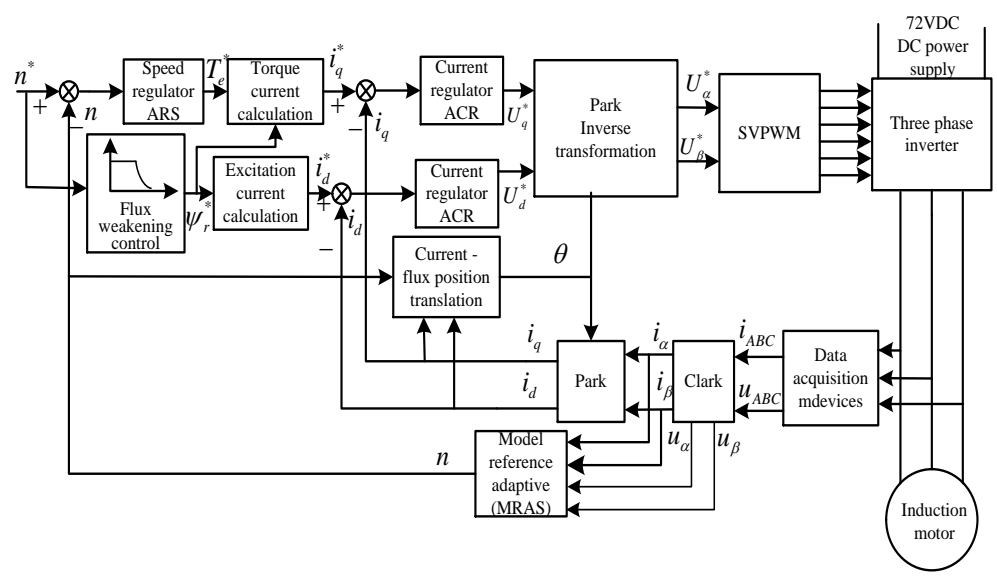

Figure 2. Speed Sensorless Vector Control System Based On Improved MRAS 


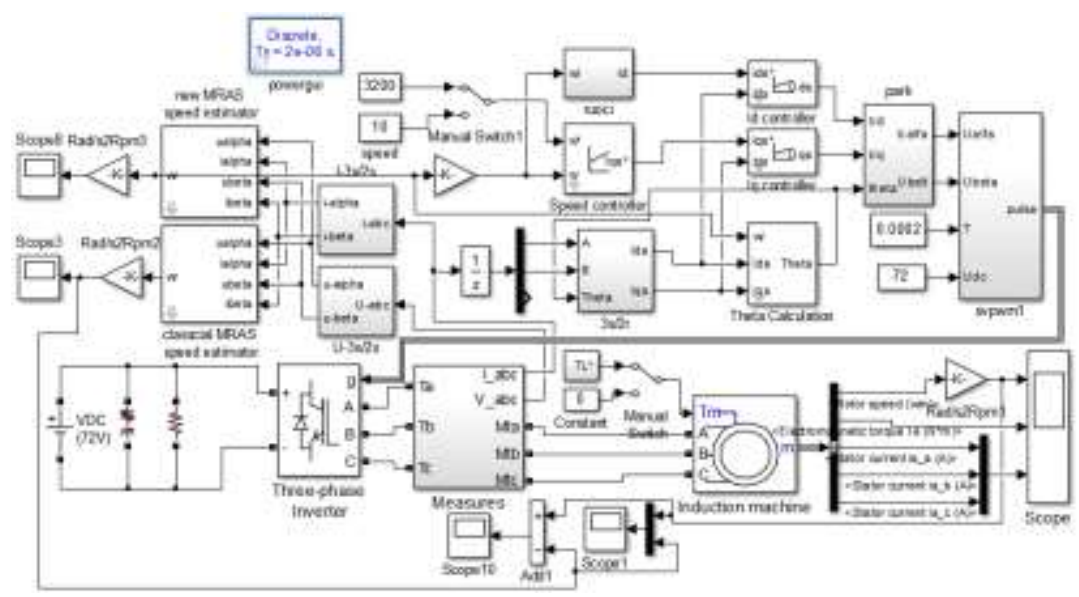

Figure 3. Simulation Model of Speed Sensorless Vector Control System Based On Improved MRAS

In order to test the accuracy of speed identification algorithm, control performance, and anti-jamming performance of induction motor in full speed range. And to compares the estimation accuracy and operational performance of the two MRAS methods at low-speed region, given the speed and load torque conditions shown in Table 1.

Table 1. Given Speed and Load Torque Condition

\begin{tabular}{|c|c|c|c|}
\hline Time (s) & $\begin{array}{c}\text { Given Speed } \\
(\mathrm{rad} / \mathrm{min})\end{array}$ & Time $(\mathrm{s})$ & $\begin{array}{c}\text { Given Load } \\
\text { torque(N.m) }\end{array}$ \\
\hline 0 & 3000 & 0 & 0 \\
\hline 0.5 & 10 & 0.5 & 8 \\
\hline 1 & 1500 & 0.7 & 0 \\
\hline
\end{tabular}

According to the simulation model of Figure 3, we can obtain the actual speed, torque, and the three-phase stator current simulation waveforms of the induction motor shown in Figure 4.
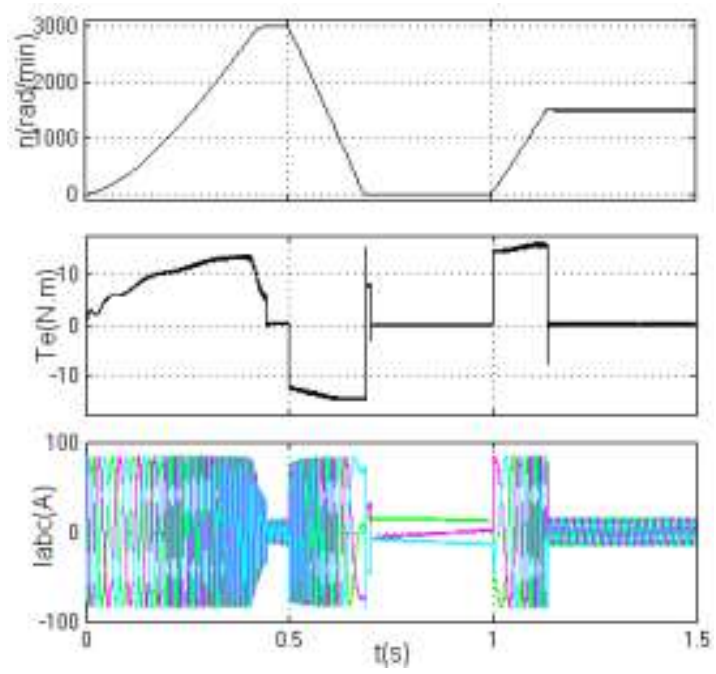

Figure 4. The Actual Simulation Waveform of the Induction Motor

From the simulation results shown in Figure 4, we can know that the starting torque of the induction motor is strong, reaction time is short, and the motor reached a steady state basically at $0.4 \mathrm{~s}$. Adding the load disturbance to motor at $0.5 \mathrm{~s}-0.7 \mathrm{~s}$ makes a little impact on 
the induction motor. The whole system has a good capacity of resisting disturbance and can response quickly.

The induction motor operates under the condition shown in Table 1, we can obtain the waveform of actual speed and the two estimated speed shown in Figure 5. The error waveform of speed estimated by traditional MRAS and the actual operating speed is shown in Figure 6, and the error waveform of speed estimated by improved MRAS and the actual operating speed is shown in Figure 7.

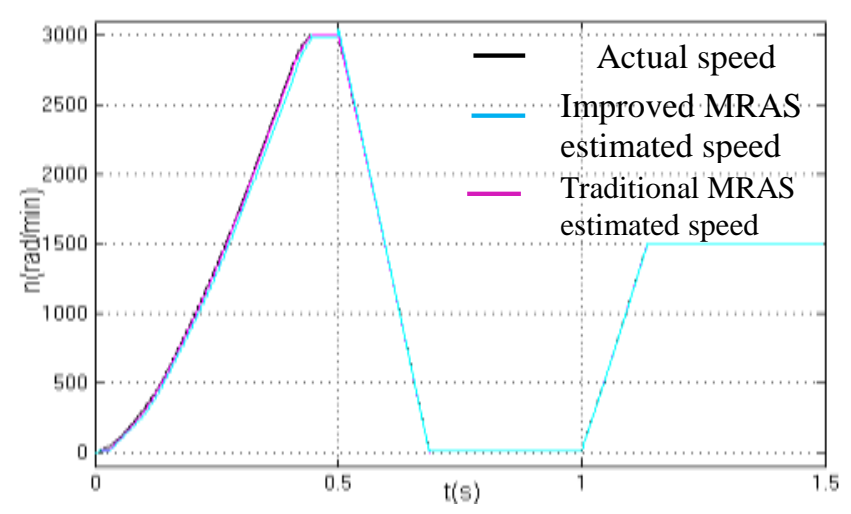

Figure 5. The Actual Speed and Estimated Speed

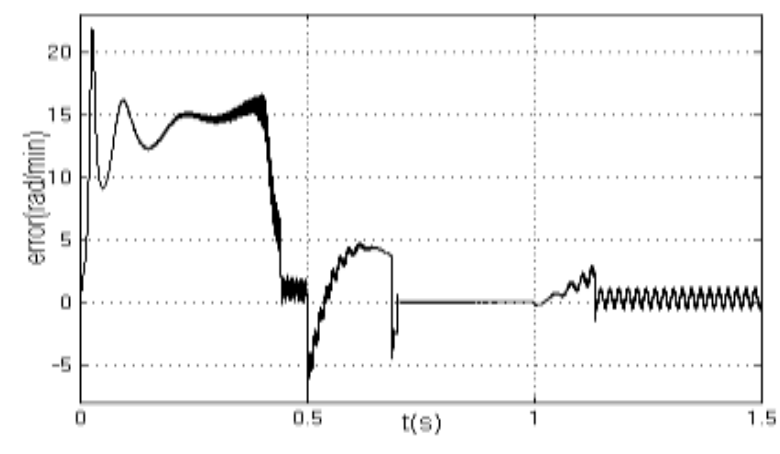

Figure 6 . The Error Waveform of Speed Estimated By Classical MRAS and Actual Speed

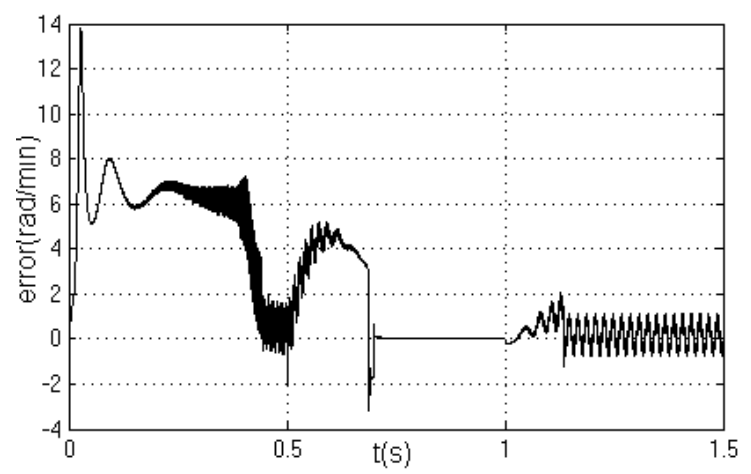

Figure 7. The Error Waveform of Speed Estimated By Improved MRAS and Actual Speed

According to the simulation results, we can be learned that use the classical MRAS and the improved MRAS speed estimator in speed sensorless vector control system of induction 
motor for electronic vehicle can obtain approximately the same accuracy of estimation at high speed and medium speed after reaching the steady state. The control system can run into a stable state quickly, and has a strong capacity of resisting disturbance. However, at low-speed region, estimation accuracy of the improved MRAS is higher than the calssical MRAS. It has a better capacity of resisting disturbance. The overall control system is more stable, and has good robustness with respect to parameter variations.

The improved MRAS method proposed above is used in the speed sensorless vector control system [15] of induction motor for electric vehicle. Use TI's DSP TMS320F1812 to control the system and then verify the feasibility of the algorithm. Detect stator current and DC voltage by Hall sensor, then input to the DSP A / D module to estimate rotor speed. Use 64 pulses / rev rotary encoder to calculate the actual speed of the motor. It's interrupts flow chart shown in Figure 8 . The rotor speed is given as $150 \mathrm{rad} / \mathrm{min}(0.05 \mathrm{pu})$ in program. The waveform of speed signal obtained by the encoder is shown in Figure 9.

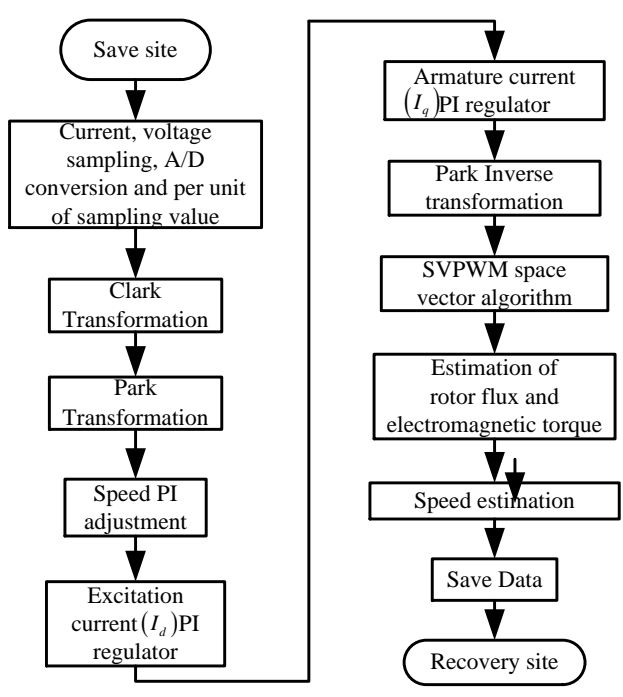

Figure 8. Interrupt Flow Chart

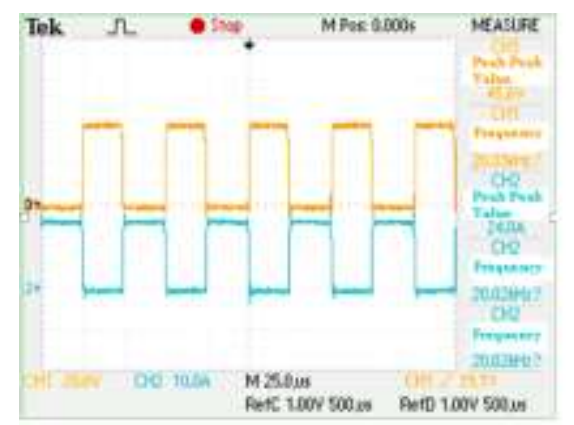

Figure 9. Waveform of Actual Speed Obtained By the Rotary Encoder

According to the actual speed waveform obtained by the rotary encoder shown in Figure 9, we know that the motor moves forward, and the speed obtained by the rotary encoder can be expressed as:

$$
\omega=60 \times \frac{1}{64 \square t}=\frac{60}{K_{p} \times T_{C L K} \times T_{\square}}=\frac{60}{64 \times 128 \times \frac{T_{\square}}{150 \times 10^{6}}}
$$

We can get the actual speed of the motor is $146.5 \mathrm{rad} / \mathrm{min}$. The induction operates smooth. 
The simulation and experimental results prove that the improved MRAS speed estimator proposed in this paper and the control strategy of the whole system are feasible. There is a good estimate accuracy of the motor at the low speed region. The static and dynamic performances of induction motor are stability.

\section{Conclusion}

This paper presents an improved MRAS speed estimation method to improve the performance of speed sensorless vector control system of induction motor for electric vehicle. Its stability can be verified by Lyapunov stability theory and Popov hyperstability theory. The simulation and experimental results show that the improved MRAS speed estimator can estimate the actual speed at low or zero speed region accurately, and the estimator has good robustness with respect to parameter variations and load torque variations at low or zero speed region. It is more suitable for electric vehicles of speed sensorless vector control than then traditional MRAS. It promotes the integration of electric vehicles and efficient development.

\section{Acknowledgements}

This work was supported by the National Natural Science Foundation of China (11472103), the key project of science and technology plan of Hunan Province (2013XK4013), the project of science and technology plan of Hunan Province (2012GK3080).

\section{Reference}

[1] X. Wen, "Present Situation and Development of Electric Motor Drive Technology in Electric Vehicle Application", Power Electronics, vol. 2, no. 3, (2013), pp. 5-8.

[2] B. Chen and G. Yang, "Three Approaches to the Control Strategies of Sensorless High-performance ASD Systems and Proposals for their Development", Electric Drive, vol. 36, no. 1, (2006), pp. 1-4.

[3] Q. L. Wang, C. Zhang and X. Zhang, "Variable-structure MRAS Speed Identification for Speed Sensorless Vector Control of Induction Motor", Proceedings of the SCEE, vol. 27, no. 15, (2007), pp. 70-72.

[4] J. Zhang, J. Chai, X. Sun and H. Lu, "An Improved Voltage Model Integral Algorithm of Induction Motors based on the Orthogonality between Back EMF and Flux", Transactions of China Electrotechnical Society, vol. 29 , no. 3, (2014), pp. 41-44.

[5] G. Wang, W. Chen, R.F. Yang, Y. Yu and D. Xu, "Improved rotor flux estimator for sensorless inductionmotor", ElectricMachines and Control, vol. 12, no. 5, (2009), pp. 638-642.

[6] B. Y. Wang and H. Feng, "Stator-current-based MRAS estimator for induction motors", Electric Machines and Control, vol. 17, no. 9, (2013), pp. 48-52.

[7] C. Schauder, "Adaptive speed identification for vector control of induction motors without rotational transducers", IEEE Trans. Ind. Appl., vol. 28, no. 5, (1992), pp. 1054-1061

[8] Y. Liao and F. Zhang, "Research of Sensorless Vector Control System and Speed Estimation", Transactions of China Electrotechnical Society, vol. 19, no. 2, (2004), pp. 36-40.

[9] B. Chen and M. Chen, "Alternating Current Drive System", Machinery Industry Press, Beijing, (2005).

[10] Q. Yu, "Research on DSP-based SVPWM vector control system for asynchronous motor", Chongqing: Chongqing University, (2010).

[11] B. Karanayil, M. F. Rahman and C. Grantham, "An implementation of a programmable cascaded low-pass filter for a rotor flux synthesizer for an induction motor drive", IEEE Trans. Power Electron, vol. 19, no. 2, (2004), pp. 257-263.

[12] M. S. Zaky, M. M. Khater, S. S. Shokralla and H. A. Yasin, "Wide speed-range estimation with online parameter identification schemes of sensorless induction motor drives", IEEE Trans. Ind. Electron, vol. 56, no. 5, (2009), pp. 1699-1707.

[13] B. Idriss, D. Said, C.-A. Larbi and O. Mohammed, "Implementation of a New MRAS Speed Sensorless Vector Control of Induction Machine", IEEE Trans. Ind. Electron, (2014), pp.1556-1563.

[14] A. R. Haron and N. R. N. Idris, "Simulation of MRAS-based Speed Sensorless Estimation of Induction Motor Drives using MATLAB/SIMULINK”, in IEEE International conference on Power and Energy, (2006), pp. 411-415.

[15] S. Rind, Y. Ren and L. Jiang, "MRAS based Speed Sensorless Indirect Vector Control of Induction Motor Drive for Electric Vehicles”, Trans. Ind. Electron, (2014), pp.978-985. 


\section{Author}

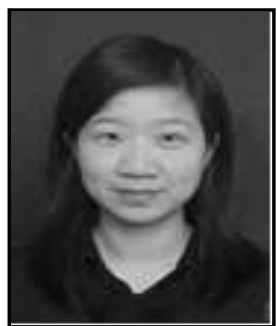

Shi Xiaorong, she is an associate professor. She received the M.Eng. from Xiangtan University in 2008. Her reserch interests are electric mearuement and control, intellgent appliances. 
International Journal of Control and Automation

Vol. 10, No. 1 (2017) 